\title{
Mathematically Modeling the Mass-Effect of Invasive Brain Tumors
}

\author{
Taylor Hines* \\ School of Mathematical and Statistical Sciences \\ Arizona State University \\ P.O. Box 871804 \\ Tempe, AZ 85287-1804
}

July 28, 2010

\begin{abstract}
When developing an accurate model of the development of glioblastomas multiforme, it is important to account not only for the invasion and diffusion of tumor cells into healthy tissue but also the resulting mass effect and brain tissue deformation. This motivates the model presented here, which implements the finite element method to solve a boundary value problem defined through classical continuum mechanics. Intended to improve existing models of tumor invasion, this model predicts the mass-effect of an invading tumor in heterogeneous brain tissue. Several parameters, taken from existing literature, dictate the behavior of differing types of brain matter. The model operates on a two-dimensional (2D) domain and outputs the displacement of brain tissue as a result of the pressure surrounding and within the tumor (peri-tumor pressure).
\end{abstract}

*taylor.hines@asu.edu

The author would like to thank Professor Eric Kostelich for his help and support throughout this project. 


\section{Introduction}

Glioblastomas Multiforme (GBMs) are aggressive, grade-4 primary brain tumors characterized by an extremely low survival rate. In fact, the median survival time of patients diagnosed with this condition is between 8 and 15 months [16]. The aggressive expansion of these tumor cells, coupled with widespread infiltration into healthy brain tissue and acutely poor vasculature, makes treatment of GBMs a delay tactic at best.

Due to the overexpression of vascular growth factors in GBMs, the vasculature of a GBM is unstructured and very permeable. This, in addition to the violent expansion of GBMs, causes massive inflammation and excessive fluid leakage, or edema. This swelling and surrounding pressure causes catastrophic and often fatal damage to neighboring brain tissue, the so-called mass-effect. It is therefore important to take this mass-effect into account when studying and predicting tumor development.

Current models of brain tumor development (e.g. [7, 15]), although accurate predictors of brain tumor development and cancer spread, largely use a geometrically static domain and therefore cannot account for the deformation of brain tissue that results from peri-tumor edema. Because the skull does not expand to accommodate a growing tumor, the mass-effect is a leading cause of morbidity from gliomas. Therefore, it is natural to consider the structural dynamics of a developing tumor. This model introduces a method to implement a model of tumor growth on a deformable domain. The model describes the dynamics in a representative 2-dimensional crosssection, as might be seen in a slice of a magnetic resonance image (MRI). Essentially, 
this project combines two separate models: one for tissue displacement and another for tumor growth. For a summary of the model results and a discussion on possible improvements, see Sections 4 and 5.

\section{The Tumor Model}

The first part of the model describes the growth dynamics of tumor cells. Because the spatial- and timescale must be large in order to model the effects of a tumor on the entire brain, only the essential characteristics of gliomas are considered. The assumptions and methods of model development and parameter selection are discussed below.

Biological Motivation. We begin by considering an (infinitesimally) small area of brain tissue $\Delta \Omega$ with sides $d x$ and $d y$, as shown in Figure 1.

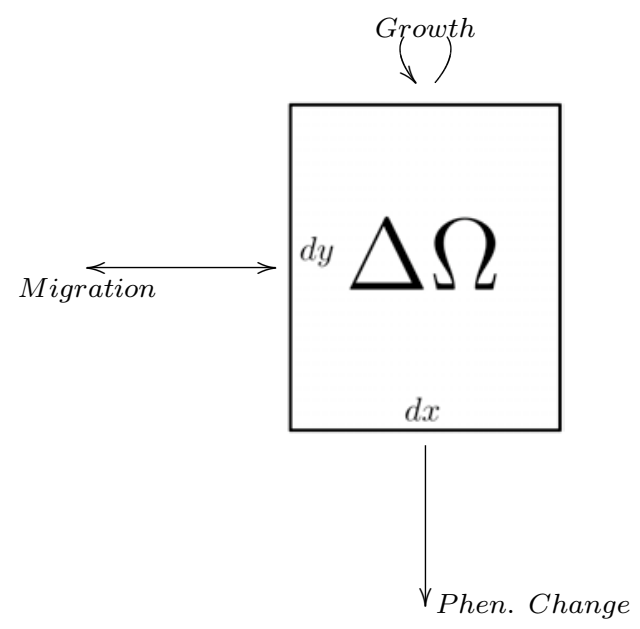

Figure 1: Two-dimensional tumor development diagram

The model presented here considers two classes of tumor cells, growing and mi- 
grating, differentiated by their patterns of behavior (phenotype). Since we partition the tumor cells by phenotype, we include phenotype changes in the conservation equations as well. As explained in Section 2, this model only considers two phenotype classes, although many different patterns of behavior may exist in actuality. Here we use only these two characteristics to formulate the two-dimensional conservation equation.

$$
\begin{aligned}
& \frac{\partial u}{\partial t}=\underbrace{D_{u}(x) \nabla^{2} u}_{\text {diffusion }}+\underbrace{\rho u\left(1-\frac{u+v}{\kappa}\right)}_{\text {logistic growth }}-\underbrace{\beta v}_{\text {class switch }} \\
& \frac{\partial u}{\partial n}=u=0 \text { on } \partial \Omega \\
& \frac{\partial v}{\partial t}=\underbrace{D_{v}(x) \nabla^{2} v}_{\text {diffusion }}+\underbrace{\beta v}_{\text {class switch }} \\
& \frac{\partial v}{\partial n}=v=0 \text { on } \partial \Omega
\end{aligned}
$$

Variables: $u(x, t)=$ Proliferating glioma cells $v(x, t)=$ Migrating glioma cells

Table 1: Equations for Tumor Development

The 'Go or Grow Hypothesis' of glioma development, under which our model operates, states that proliferation and migration are mutually exclusive in tumor cells. According to this hypothesis, tumor cells are characterized by one of these two phenotypes.

This hypothesis is not universally accepted, and several studies have produced evidence against it (see [5]). Arguments against the Go or Grow Hypothesis primarily 
show that it can lead to unreliable results at the micro level. However, mathematical models of tumor development based on the Go or Grow hypothesis (e.g. [7, 19]) have achieved numerically accurate results, and it is believed to be an accurate predictor of glioma behavior at the macro level. For a concise history, as well as a summary of recent biological research relating to migration and proliferation in gliomas, see [1]. In this model, we treat migrating and proliferating tumor cells as completely distinct, and they are modeled as different variables.

Growth. Equation (1) describes the dynamics of the variable $u$, which gives the number of proliferating tumor cells throughout the domain. Although these cells are assumed not actively to migrate throughout the brain, we include an undirected diffusion term to represent random motion. Therefore, the coefficient of diffusion $D_{u}$ is very low. However, $D_{u}$ varies according to tissue type to account for the fact that some brain tissue is more permeable than other tissue types (e.g. white vs. gray matter). Since tumor cells reproduce exponentially until they reach carrying capacity, Equation (1) also contains a logistic growth term with carrying capacity $\kappa$ and growth rate $\rho$. These parameters are assumed not to vary throughout the domain and are gathered from existing literature (see Appendix A). The final term in this equation describes the loss of cells due to phenotype change. Note that this is an approximation, since the assumption of a constant class switch is known to be inaccurate; see Section 5 for further discussion.

Diffusion and Invasion. Equation (3) describes the dynamics of the variable $v$, which gives the number of migrating cells throughout the domain. The behavior of these cells is modeled with an diffusion term with spatially varying coefficient of 
diffusion $D_{v}$. Because invasion and diffusion is so much more aggressive in migrating cells (see Appendix A), we set $D_{v}$ much greater than $D_{u}$. Both $D_{u}$ and $D_{v}$ are very small in regions corresponding to cerebrospinal fluid (CSF), reflecting the very small diffusion of tumor in this region. The second term in this equation represents the net gain due to phenotype switch from proliferating cells.

Boundary Conditions. Equations (2) and (4) represent the mixed Dirichlet and Neumann boundary conditions of the skull. We assume that the skull is completely impermeable, so that no tumor cells can migrate through it. In addition, we assume that it is impossible for tumor cells to grow within the skull, so the tumor density is fixed at zero there. We also assume that such a boundary completely surrounds the brain, which is an assumption that may be relaxed in the future (i.e., allowing glioma cells to migrate out through the bloodstream or the spinal column).

Continuum Assumption. Since this is a macro-scale model, we model the brain as a continuum. To reflect physical reality, the domain is segmented into several tissue types, namely white and gray matter, CSF, the falx cerebri, and the skull. The permeability of each tissue type and other parameters are taken from existing literature and are varied throughout the domain (see Appendix A).

Our domain was adapted from those made available through the BrainWeb Database [4]. The high level of detail available from this database made possible a spatially accurate model of brain geometry, which is especially important when developing a physical model of tumor mass-effect. 


\subsection{The Mass-Effect Model}

To model the mass-effect that occurs as a tumor develops, we also define a biomechanical elasticity problem that dictates how the domain will shift with tumor growth. Unlike the model for the growth of tumor cells, this system is not time-dependent, although the boundary conditions change as the tumor grows. Instead, we assume that tumor mass-effect can be modeled as a quasi steady-state. Due to the relatively slow growth of a tumor (characteristic timescale $\sim 1$ day) we do not expect a relaxation of this assumption to produce better results. Essentially, this problem reduces to calculating the strain $\epsilon$ experienced by the tissue in response to the stress $\sigma$ due to peri-tumor edema.

Physical Motivation. We start by considering an infinitesimal rectangular area of tissue $\Delta \Omega$, with sides $d x$ and $d y$. The forces acting upon $\Delta \Omega$ are the normal stresses $\sigma_{x}$ and $\sigma_{y}$, which act perpendicularly to $d y$ and $d x$, respectively. In addition, there is a shear stress $\tau_{x y}\left(\tau_{y x}\right)$ that acts on $d x(d y)$ but in a parallel direction. These stresses are shown in Figure 2.

In response to these stresses, the tissue experiences movement, known as strain. Similarly to the stress vectors, the strain has components $\epsilon_{x}, \epsilon_{y}$ and $\gamma_{x y}, \gamma_{y x}$ known as the normal strains and shear strains, respectively.

Model Assumptions. The relationship between the stress and strain experienced by a material is known as the constitutive equation, which depends greatly on the type of tissue being studied. In this simulation, we simplify by assuming that brain tissue is a linear elastic solid that behaves according to Hooke's Law. Under 


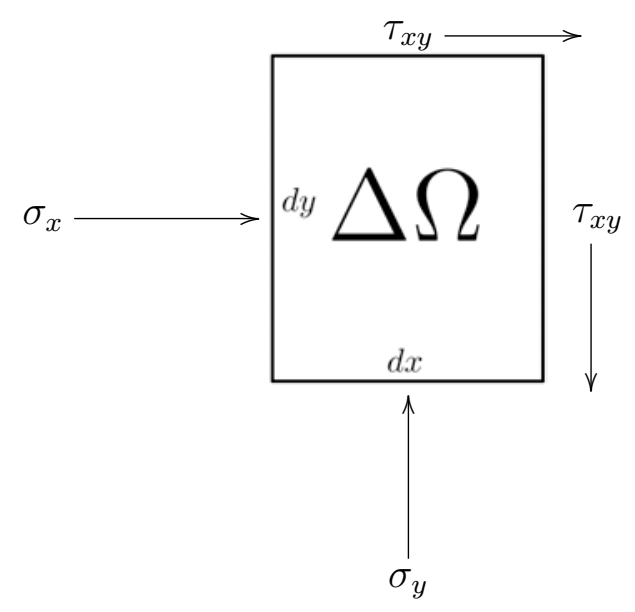

Figure 2: Two-dimensional stress diagram

this assumption, the constitutive equation is linear and is given explicitly by

$$
\left(\begin{array}{c}
\sigma_{x} \\
\sigma_{y} \\
\tau_{x y}
\end{array}\right)=\frac{E}{1-\nu^{2}}\left(\begin{array}{ccc}
1 & \nu & 0 \\
\nu & 1 & 0 \\
0 & 0 & \frac{1-\nu}{2}
\end{array}\right)\left(\begin{array}{c}
\epsilon_{x} \\
\epsilon_{y} \\
\gamma_{x y}
\end{array}\right)
$$

The parameter $E$ is the Young's modulus, which gives the stiffness of elastic material, and $\nu$ is the Poisson's ratio, which governs compressibility. For a full derivation of these equations, see $[9,11]$. The stiffness and compressibility of each tissue type is varied throughout the domain, depending on tissue type (see Appendix A).

Boundary Conditions. The boundary (skull) is assumed to be immobile. In addition, we apply a no-slip condition to the brain tissue near the skull, because there are unseen physiological structures that keep the brain in place.

The mass-effect model is run in between each timestep of the cell-growth model. The initial force on the brain tissue at each timestep is applied in proportion to the 
density of tumor cells at each location and in the direction of the gradient of this density. Although this method applies force in the correct direction, it is somewhat ad-hoc and a better method of force application is in development (see Section 5). This model does not account for the force on the brain tissue due to gravity. Once these initial conditions are set, the resulting displacement is calculated numerically.

\section{Numerical Solution}

The systems defined above are solved numerically using finite element analysis. In short, we wish to approximate the exact analytical solution with a piecewise linear function. Integrating then gives us a linear system that can be easily solved on a computer. In the following sections, we review only the solution of the system that defines tumor growth and development. The solution of the biomechanical problem is similar and in many ways simpler, since it is the solution of a linear system, rather than several systems of partial differential equations. For more detail about the formulation and solution of the mass-effect model, see $[9,11]$.

\subsection{Variational Formulation}

In order to solve the system numerically, we must first find the variational (or weak) formulation of the system of partial differential equations defined above. For example, if $u$ is a solution to the equation

$$
\frac{\partial u}{\partial t}=D_{u}\left(\frac{\partial^{2} u}{\partial x^{2}}+\frac{\partial^{2} u}{\partial y^{2}}+\beta u\right)
$$


then it follows that

$$
\int_{\Omega} w \frac{\partial u}{\partial t} d \Omega=D_{u} \int_{\Omega}\left(w \frac{\partial^{2} u}{\partial x^{2}}+w \frac{\partial^{2} u}{\partial y^{2}}+\beta w u\right)
$$

for any function $w$ satisfying the same boundary conditions. This formulation allows us to integrate by parts. Given the no-flux condition on the boundary (skull), we can use Green's Theorem to achieve the following form of Equations (6) and (7):

$$
\begin{aligned}
\int_{\Omega} w \frac{\partial v}{\partial t} d \Omega & =D_{v} \int_{\Omega}\left(\frac{\partial w}{\partial x} \frac{\partial v}{\partial x}+\frac{\partial w}{\partial y} \frac{\partial v}{\partial y}\right) d \Omega \\
& +\rho \int_{\Omega} w v\left(1-\frac{u+v}{\kappa}\right) d \Omega-\beta \int_{\Omega} w u d \Omega \\
\int_{\Omega} w \frac{\partial u}{\partial t} d \Omega & =D_{u} \int_{\Omega}\left(\frac{\partial w}{\partial x} \frac{\partial u}{\partial x}+\frac{\partial w}{\partial y} \frac{\partial u}{\partial y}\right) d \Omega+\beta \int_{\Omega} w u d \Omega
\end{aligned}
$$

Now, rather than finding the solution $u$ (or $v$ ) for all functions $w$, we numerically find a solution $u$ with $w$ ranging only over a finite-dimensional family. ${ }^{1}$

\subsection{Discretization}

As stated above, we wish to approximate the solutions $u$ and $v$ with functions $\tilde{u}$ and $\tilde{v}$ that are piecewise linear. This requires dividing the domain into a finite number of elements. ${ }^{2}$ Since there exist efficient algorithms for Delaunay triangulation of the

\footnotetext{
${ }^{1}$ For more information on the variational form and the finite element method, see e.g. [11].

${ }^{2}$ Since $\tilde{u}$ is linear on each element, we can clearly see that the accuracy of the approximate solution grows as the number of elements increases. This must be balanced against the computational power at hand. In this case, we use a relatively coarse domain (on the order of $10^{4}$ elements).
} 
plane, (see, e.g. [18]) and because linear interpolation on three points is so simple, we use triangular elements for this project. To divide the domain into elements, we first place nodes at regular intervals throughout the domain. Then, using the program Triangle [18], these nodes are connected into a mesh of equal sized, non-overlapping triangles that partition the domain. Note that these elements do not remain equally sized, since the domain moves as the tumor expands.

The same mesh is used to solve the reaction-diffusion equation as is used to define the $2 \mathrm{D}$ elastic elements in the displacement equation. Although this is different than most other projects, when an interface is defined between moving surfaces, this method was used deliberately. Because tumors are so diffuse and invasive, it is literally impossible to define an interface between healthy and cancerous tissue. Therefore, some traditional methods of continuum mechanics fail since there is not a well-defined boundary where motion occurs. This approach raises several problems. Most notably, the elements where tumor cell density is high expand as the tumor does (due to the fact that force is being applied outwardly), which significantly lowers the resolution of the approximate solution. To show convergence of the numerical solution, we must constantly remesh by adding more triangular elements as those beneath the tumor grow out of proportion, which can pose quite a challenge. However, in this project, we are instead searching for only qualitatively good results, and therefore these problems have not yet been considered. 


\subsection{Finite (linear) approximation}

To approximate the exact solution of the model, we represent the solutions $u$ and $v$ with a finite basis of compactly supported linear functions, which allows us to integrate the system numerically. Since we wish the approximate solutions $\tilde{u}$ and $\tilde{v}$ to be linear on each triangular element, we choose this basis so that three basis functions are supported on each element, representing linear interpolation of the solution. That is, we define the functions

$$
\begin{aligned}
f_{1}(x, y) & =\frac{1}{2 A}\left(\left(b_{1} c_{2}-c_{1} b_{2}\right)+\left(b_{2}-c_{2}\right) x+\left(c_{1}-b_{1}\right) y\right) \\
f_{2}(x, y) & =\frac{1}{2 A}\left(\left(c_{1} a_{2}-a_{1} c_{2}\right)+\left(c_{2}-a_{2}\right) x+\left(a_{1}-c_{1}\right) y\right) \\
f_{3}(x, y) & =\frac{1}{2 A}\left(\left(a_{1} b_{2}-b_{1} a_{2}\right)+\left(a_{2}-b_{2}\right) x+\left(b_{1}-a_{1}\right) y\right)
\end{aligned}
$$

on each element, where $a=\left(a_{1}, a_{2}\right), b=\left(b_{1}, b_{2}\right)$, and $c=\left(c_{1}, c_{2}\right)$ are the three element vertices and $A$ is the element area. Note that these satisfy the condition that if $d_{j}$ is one of the vertices $a, b$, or $c$, then $f_{i}\left(d_{j}\right)=\delta_{i, j}$, the Kronecker Delta

function. Furthermore, $\sum_{i=1}^{3} f_{i}=1$ as desired. Therefore, on each element $e$, we use the approximate linear solutions

$$
\tilde{u}_{e}=\sum_{i=1}^{3} f_{i}(x, y) u_{i} \quad \text { and } \quad \tilde{v}_{e}=\sum_{j=1}^{3} f_{j}(x, y) v_{j}
$$


where $u_{i}$ and $v_{i}$ is the value of $u$ and $v$ at node $i$. Generalizing to the entire domain, and with a slight abuse of notation, we use the global approximations

$$
\begin{aligned}
\tilde{u} & =\sum_{e=1}^{n} \sum_{i=1}^{3} f_{e, i}(x, y) u_{e, i} \\
\tilde{v} & =\sum_{e=1}^{n} \sum_{j=1}^{3} f_{e, j}(x, y) v_{e, j},
\end{aligned}
$$

where $f_{e, i}$ is the function $f_{i}$ local to element $e, u_{e, i}$ is the value of $u$ at node $i$ on element $e$, and $n$ is the total number of elements. ${ }^{3}$ We then substitute these approximate solutions for $u$ and $v$ in Equations (8) and (9) as defined in Section 3.1.

Recall from Section 3.1 that $w$ ranges over a finite-dimensional family of functions defined on the domain. Here, we use

$$
w=\sum_{e=1}^{n}\left(\begin{array}{c}
f_{e, 1}(x, y) \\
f_{e, 2}(x, y) \\
f_{e, 3}(x, y)
\end{array}\right)
$$

with the same notation as in Equations (13) and (14).

Integrating in both spatial dimensions (not time) then gives us the following linear

\footnotetext{
${ }^{3}$ It is important to note that there is some overlap here, that is, there will be many integers $i \neq j$ or elements $e \neq f$ such that the element-node pair $(e, i)$ and $(f, j)$ refer to the same node. This is because many elements may share the same vertices.
} 
system of ordinary differential equations: ${ }^{4}$

$$
\begin{aligned}
{[M][\dot{v}]^{t} } & =\left[K_{v}\right][v]^{t}-\rho[M]\left[v\left(1-\frac{v+u}{\kappa}\right)\right]^{t}-\beta[M][u]^{t} \\
{[M][\dot{u}]^{t} } & =\left[K_{u}\right][u]^{t}+\beta[M][u]^{t} .
\end{aligned}
$$

For a full derivation of this system, see Appendix B.

\subsection{Linear solution}

Now that we have converted the system defined in Section 2 to a system of ordinary differential equations, we can find a solution using any number of ODE methods. In this case, we use the backward Euler method. As explained in Section 5, a linear model of tissue deformation is not considered inaccurate (as shown in [13]), but this simplified model is used here for simplicity while a more complex model is in development. The resulting linear system is solved on a computer with the aid of LAPACK [2]. All other computations and preprocessing were performed with Fortran 90. As mentioned in Section 3.2, mesh generation and visualization were performed with the aid of Triangle [18]. Due to the banded nature of the shape and stiffness matrices, this system can be solved relatively quickly.

\footnotetext{
${ }^{4}$ Note that arithmetic operations are performed elementwise in Equation (16).
} 


\section{Results}

The model begins with a tumor 'seed,' a small $\left(\sim 10^{-2}\right.$ in $\left.^{2}\right)$ area of the domain where the density of tumor cells is set very high (to carrying capacity). The tumor then grows and invades according to the reaction-diffusion equations defined in Section 2. At each timestep, the number of tumor cells as well as the gradient of cells density is calculated at every node. We then calculate the resulting force at each node, in proportion to the number of cells at each node, and in the direction of that gradient. These force vectors are then treated as the initial conditions of the elasticity problem defined in Section 2.1, which gives the strain at each node. The nodes are then moved accordingly, and then the domain is remeshed. If desired, the output is saved as an image file. The model is usually run with a timestep of one day and for a duration of 10-14 months.

Although numerical convergence of our results was not tested, we were successful in producing results that compared qualitatively with MRI images. Specifically, characteristic behavior such as collapse of the ventricles and bending of the falx cerebri was predicted and was similar to observed data. Figure 3 is a sample output of the model.

Even though the model was not designed to imitate this particular patient case, notice that they share many qualitative similarities. Most notably, the left ventricle has been pushed up and to the left, while the right ventricle has almost completely collapsed. In addition, notice that the lower right lobe has been pushed out of shape, and the membrane dividing the left and right hemispheres (the falx) has been bowed 

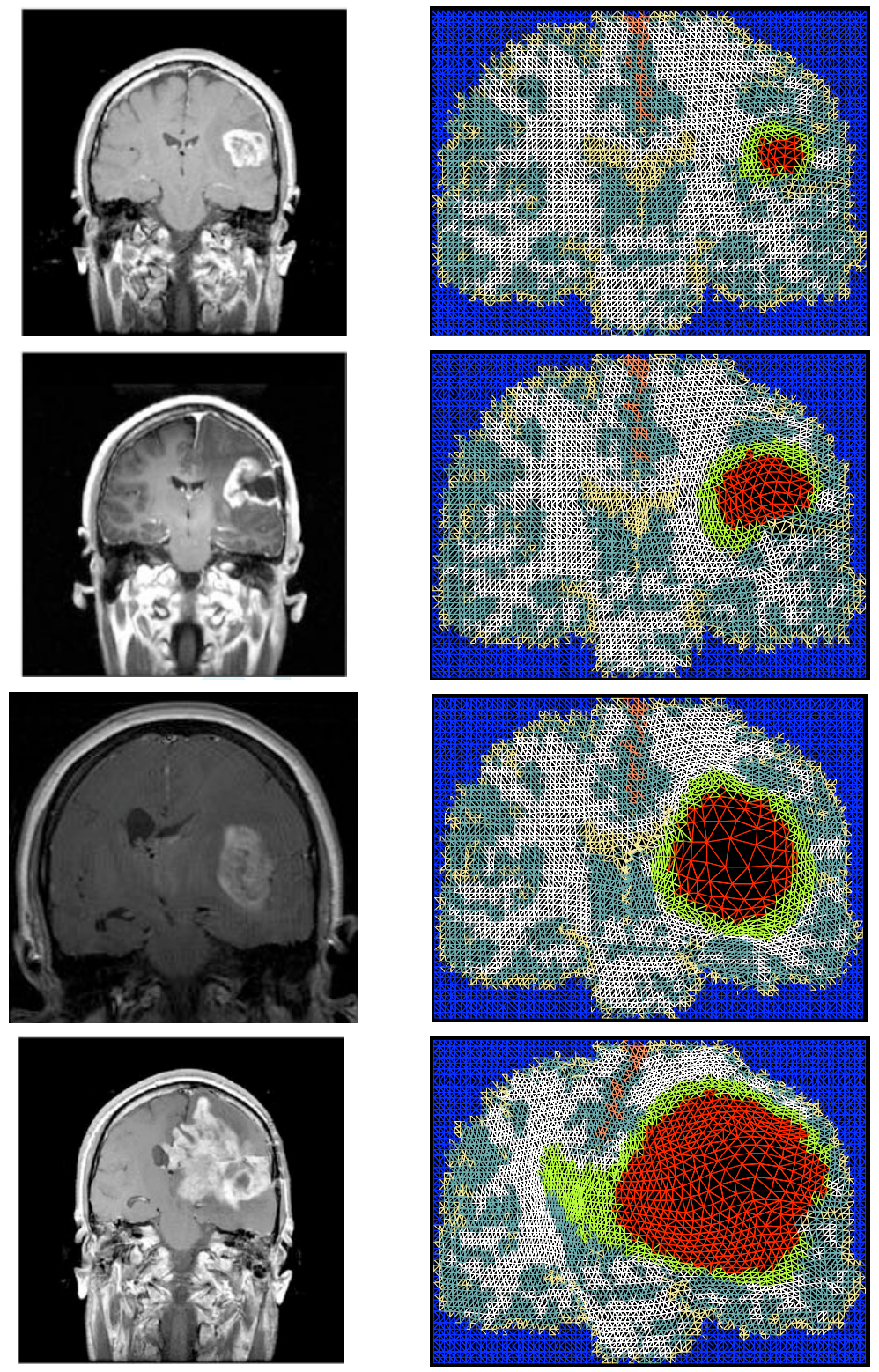

Figure 3: Sample model output.

slightly to the left. All of this is due to the pressure from the expanding tumor, and this behavior has been captured in the tumor model. 
This model is still far from a quantitatively accurate predictor of glioma development, but initial results appear promising. Specifically, the model of tumor masseffect used here is not tied to any single model of tumor growth, and therefore a different model of tumor development can be used if it is observed to produce more accurate results. For a discussion of future work and other improvements that may be integrated into this model, see Section 5.

\section{Remarks}

As stated in Section 2, the model implemented here is only an initial approximation of tumor behavior. In order to model the behavior of gliomas with more accuracy, it is necessary to account for a multitude of other factors. Future areas of improvement include, but are definitely not limited to, the dramatic importance of vascular development, the various growth factors that motivate the growth and development of tumor cells and associated vasculature, the tumor's interaction with and subsequent degradation of the extracellular matrix (ECM), the effects of growth, migration and death due to crowding, and the effects of chemotherapy, radiotherapy, and other forms of treatment.

Perhaps the most important area for development is to model the mass-effect that occurs due to brain surgery, most importantly the resection of a developing tumor. First of all, since the effects of gravity are not considered in the current model of tumor mass-effect, we must develop a method of modeling the collapse of the resection cavity after surgery. Furthermore, due to the fact that a linear model of tissue deformation 
has been shown to be inaccurate [13], a better model of brain deformation needs to be implemented in general. Inaccuracy due to a simplified, linear model of cellular deformation can be ignored, at least in early tumor development, because the extent of the mass-effect is relatively small. However, after the resection of even a small tumor, the patient's brain experiences dramatic deformation in a very short time as the resection cavity collapses. Therefore, we expect that a linear model of brain tissue deformation will not provide an accurate method of modeling such an event.

Another potential area of improvement is tumor cell phenotype switch. For simplicity, the model presented here treats this as a constant, yet it is believed that in reality, phenotype switch of tumor cells occurs stochastically as well as in response to crowding and other factors. As the density of proliferating cells reaches carrying capacity, we expect the rate of conversion to migrating cells to increase. As mentioned in Section 2, the Go or Grow Hypothesis has been relatively common in macro-level models of tumor development since 1997, but the assumption that growth and migration in tumor cells is mutually exclusive is not universally accepted. Nevertheless, models such as [7] have achieved numerically accurate results with a stochastic model of phenotype change. Either way, this aspect of our model is in need of investigation.

Finally, it is expected that a finer mesh will increase resolution and hence accuracy. We have developed a much more detailed domain, yet the code we have written to calculate the finite element solution cannot yet be run on parallel processors (is not yet MPI-aware). A version of this code is under development, which will make it possible to calculate a better-resolution numerical solution where numerical convergence of our results can be tested. 


\section{Acknowledgments}

This paper was created as part of the Computational Science training for Undergraduates in the Mathematical Sciences (CSUMS) project at Arizona State University, * and supported in part by NSF-MPS-DMP Award 0703587. All MRI images and patient case data were made available through the Barrow Neurological Institute of St. Joseph's Hospital and Medical Center in Phoenix, Arizona. † Testing and development of our research code was performed in part on the Saguaro Cluster, available through the Fulton High Performance Computing Initiative at ASU. ${ }^{\ddagger}$ This research was submitted as an undergraduate honors thesis to the Barrett Honors College at Arizona State University. $§$ A portion of this work was presented at the SIAM Conference on Computational Science and Engineering in Miami, Florida in March 2009, as well as at the Southwestern Undergraduate Mathematics Research Conference (SUnMaRC) in Albuquerque, New Mexico in February, 2009.

\section{References}

[1] V. Amberger-Murphy, Glioma invasion: mechanism, modulation and future possibilities (Editorial), Acta Neurochirurgica. 145 (2003), 613-614.

[2] E. Anderson, Z. Bai, C. Bischof, S. Blackford, J. Demmel, J. Dongarra, J. Du Croz, A. Greenbaum, S. Hammarling, A. McKenney, D. and Sorensen, LAPACK Users' Guide - 3rd ed. Society for Industrial and Applied Mathematics (1999).

[3] M. Cameron and A. Whitmer, Angiogenesis in Glioblastomas Multiforme, Available online at http://math.asu.edu/CSUMS

*For more information contact the program coordinators; Eric Kostelich (Kostelich@asu.edu) and Bruno Welfert (Welfert@asu.edu), or visit the Web page http://math.asu.edu/CSUMS/

†http://www.thebarrow.org/index.htm

${ }^{\ddagger}$ http://hpc.fulton.asu.edu/

${ }^{\S}$ http: //honors.asu.edu/index.aspx 
[4] C.A. Cocosco, V. Kollokian, R.K.-S. Kwan, and A.C. Evans, BrainWeb: Online Interface to a 3D MRI Simulated Brain Database, NeuroImage. 5(4) part 2 of 4, S425 (1997). - Proceedings of 3-rd International Conference on Functional Mapping of the Human Brain, Copenhagen, May 1997.

Database located at http://www.bic.mni.mcgill.ca/brainweb/

[5] A. Corcoran, R.F. Del Maestro Testing the "go or grow" hypothesis in human medulloblastoma cell lines in two and three dimensions, Neurosurgery. 53 (2003), 174-184.

[6] I. Doltsinis, Large Deformation Processes of Solids. WIT Press (2004).

[7] S. Eikenberry, T. Sankar, M. C. Preul, E. J. Kostelich, C. Thalhauser, and Y. Kuang, The Virtual Glioblastoma: Growth, Migration, and Treatment in a Three-Dimensional Mathematical Model, Cell Proliferation, to appear.

[8] C. Hogea, G. Biros, F. Abraham, and C. Davatzikos, A robust framework for soft tissue simulations with application to modeling brain tumor mass effect in $3 D M R$ images, Physics in Medicine and Biology 52 (2007), 6893-6908.

[9] Y.W. Kwon and H. Bang, The Finite Element Method using MATLAB. CRC Press (1997).

[10] S.K. Kyriacou, C. Davatzikos, S.J. Zinreich, and R.N. Bryan, Nonlinear Elastic Registration of Brain Images with Tumor Pathology Using a Biomechanical Model, IEEE Transactions On Medical Imaging. 187 (1999), 580-592.

[11] D.L. Logan, A First Course in the Finite Element Method. Brooks/Cole (2002).

[12] MATLAB 7.5.0.338 (R2007b), The MathWorks. (1984)

[13] K. Miller, K. Chinzei, Mechanical properties of brain tissue in tension, Journal of Biomechanics. 35 (2002), 483-490.

[14] A. Mohamed and C. Davatzikos, Finite Element Modeling of Brain Tumor Mass-Effect from $3 D$ Medical Images (MICCAI), 26-29 (2005), 400-408.

[15] J.D. Nagy, Competition and Selection in a Mathematical Model of Cancer, Bulletin of Mathematical Biology.66 (2004), 663-687.

[16] A.D. Norden and P.Y. Wen, Glioma Therapy in Adults, Neurologist.12 (2006), 279-292.

[17] J.S. Ruan, T. Khalil, and A.I. King, Dynamic Response of the Human Head to Impact by Three-Dimensional Finite Element Analysis, Journal of Biomechanical Engineering 45 Vol. 116 (1994), 44-50.

[18] J. Shewchuk, Triangle: Engineering a 2D Quality Mesh Generator and Delaunay Triangulator, Applied Computational Geometry: Towards Geometric Engineering. 1148 (1996), 202-222. Availible online at http://www.cs.cmu.edu/ quake/triangle.html 
[19] A.M. Stein, T. Demuth, D. Mobley, M. Berens, L. Sander A mathematical model of glioblastoma tumor spheroid invasion in a 3d in vitro experiment Biophys. J. 92 (2006), 356-365.

[20] A. Wasserman, R. Acharya, C. Sibata, and K.H. Shin A Patient-Specific In Vivo Tumor Model, Mathematical Biosciences. 136 (1996) 111-140.

\section{Appendices}

\section{A Physical Parameters}

\begin{tabular}{|c|c|c|c|c|}
\hline Parameter & Value & Description & Units & Source \\
\hline$E_{W M}$ & 0.29008 & Young's modulus of white matter & $\mathrm{lb} / \mathrm{in}^{2}$ & [8] p.6903 \\
\hline$\nu_{W M}$ & 0.45000 & Poisson's ratio of white matter & None & [8] p.6903 \\
\hline$E_{G M}$ & 0.36260 & Young's modulus of grey matter & $\mathrm{lb} / \mathrm{in}^{2}$ & [8] p.6903 \\
\hline$\nu_{G M}$ & 0.48500 & Poisson's ratio of grey matter & None & [14] p.404 \\
\hline$E_{C S F}$ & 0.07250 & Young's modulus of spinal fluid & $\mathrm{lb} / \mathrm{in}^{2}$ & [8] p.6903 \\
\hline$\nu_{C S F}$ & 0.48900 & Poisson's ratio of spinal fluid & None & {$[17]$ p. 45} \\
\hline$E_{\text {Falx }}$ & 14.5040 & Young's modulus of falx & $\mathrm{lb} / \mathrm{in}^{2}$ & [10] p.581 \\
\hline$\nu_{\text {Falx }}$ & 0.50000 & Poisson's ratio of falx & None & Inc. ${ }^{a}$ \\
\hline$E_{G B M}$ & 0.08363 & Young's modulus of tumor & $\mathrm{lb} / \mathrm{in}^{2}$ & Exp. ${ }^{b}$ \\
\hline$\nu_{G B M}$ & 0.48500 & Poisson's ratio of tumor & None & [14] p.404 \\
\hline$D_{v, W M}$ & $3.10 \times 10^{-6}$ & Diff. of proliferating cells in WM & $\mathrm{in}^{2} /$ day & [7] p.16 \\
\hline$D_{v, G M}$ & $6.20 \times 10^{-7}$ & Diff. of proliferating cells in GM & $\mathrm{in}^{2} /$ day & [7] p.16 \\
\hline$D_{v, C S F}$ & $1.55 \times 10^{-7}$ & Diff. of proliferating cells in CSF & $\mathrm{in}^{2} /$ day & [7] p.16 \\
\hline$D_{u, W M}$ & $1.55 \times 10^{-4}$ & Diff. of migrating cells in WM & $\mathrm{in}^{2} /$ day & [7] p.16 \\
\hline$D_{u, G M}$ & $3.10 \times 10^{-5}$ & Diff. of migrating cells in GM & $\mathrm{in}^{2} /$ day & [7] p.16 \\
\hline$D_{u, C S F}$ & $1.55 \times 10^{-7}$ & Diff. of migrating cells in CSF & $\mathrm{in}^{2} /$ day & [7] p.16 \\
\hline$\rho$ & 0.200 & Growth rate of of proliferating cells & $1 /$ day & [7] p.16 \\
\hline$\kappa$ & $6.45 \times 10^{6}$ & Glioma carrying capacity & cells/in ${ }^{2}$ & [7] p.16 \\
\hline$\beta$ & $10^{-4}$ & Rate of phenotype switch & $1 /$ in $^{2}$ & [7] p.16 \\
\hline
\end{tabular}

${ }^{a}$ We assume that this tissue is incompressible

${ }^{b}$ This parameter is experimentally approximated using numerical results. 


\section{B Derivation of Stiffness and Shape Matrices}

As summarized in Sections 3.1 and 3.3, we have the following weak formulation of a system of partial differential equations, which we hope to solve for $u$ and $v$

$$
\begin{aligned}
\int_{\Omega} w \frac{\partial v}{\partial t} d \Omega & =D_{v} \int_{\Omega}\left(\frac{\partial w}{\partial x} \frac{\partial v}{\partial x}+\frac{\partial w}{\partial y} \frac{\partial v}{\partial y}\right) d \Omega \\
& +\rho \int_{\Omega} w v\left(1-\frac{u+v}{\kappa}\right) d \Omega-\beta \int_{\Omega} w u d \Omega \\
\int_{\Omega} w \frac{\partial u}{\partial t} d \Omega & =D_{u} \int_{\Omega}\left(\frac{\partial w}{\partial x} \frac{\partial u}{\partial x}+\frac{\partial w}{\partial y} \frac{\partial u}{\partial y}\right) d \Omega+\beta \int_{\Omega} w u d \Omega .
\end{aligned}
$$

Recall that $w$ can be any function that satisfies the same boundary conditions as $u$ and $v$, namely, $u=v=\frac{\partial u}{\partial n}=\frac{\partial v}{\partial n}=0$. To integrate this system numerically, we represent the exact solutions $u$ and $v$ with respect to a finite basis of compactly supported linear functions.

We begin by restricting our attention to a single triangular element $\Omega_{e}$, and later we will generalize to the entire domain. We choose these basis functions to represent linear interpolation of the solution. That is, we define the three functions

$$
\begin{aligned}
& f_{1}(x, y)=\frac{1}{2 A}\left(\left(b_{1} c_{2}-c_{1} b_{2}\right)+\left(b_{2}-c_{2}\right) x+\left(c_{1}-b_{1}\right) y\right) \\
& f_{2}(x, y)=\frac{1}{2 A}\left(\left(c_{1} a_{2}-a_{1} c_{2}\right)+\left(c_{2}-a_{2}\right) x+\left(a_{1}-c_{1}\right) y\right) \\
& f_{3}(x, y)=\frac{1}{2 A}\left(\left(a_{1} b_{2}-b_{1} a_{2}\right)+\left(a_{2}-b_{2}\right) x+\left(b_{1}-a_{1}\right) y\right)
\end{aligned}
$$

that are only supported in $\Omega_{e}$, where $a=\left(a_{1}, a_{2}\right), b=\left(b_{1}, b_{2}\right)$, and $c=\left(c_{1}, c_{2}\right)$ are the three element vertices and $A$ is the element area.

Note that these satisfy the condition that if $d_{j}$ is one of the vertices of $\Omega_{e}$, then $f_{i}\left(d_{j}\right)=\delta_{i, j}$, the Kronecker Delta function. Furthermore, $\sum_{i=1}^{3} f_{i}=1$ as desired. ${ }^{5}$ Therefore, we use the approximate linear solutions

$$
\tilde{u}=\sum_{i=1}^{3} f_{i}(x, y) u_{i} \quad \text { and } \quad \tilde{v}=\sum_{j=1}^{3} f_{j}(x, y) v_{j},
$$

${ }^{5}$ This is easy to check, due to the fact that $A=\frac{1}{2} \operatorname{det}\left(\begin{array}{ccc}1 & a_{1} & a_{2} \\ 1 & b_{1} & b_{2} \\ 1 & c_{1} & c_{2}\end{array}\right)$. 
where $u_{i}$ and $v_{i}$ is the value of $u$ and $v$ at node $i$. More simply,

$$
\begin{gathered}
\tilde{u}=\left(\begin{array}{lll}
f_{1} & f_{2} & f_{3}
\end{array}\right)\left(\begin{array}{l}
u_{1} \\
u_{2} \\
u_{3}
\end{array}\right) \\
\tilde{v}=\left(\begin{array}{lll}
f_{1} & f_{2} & f_{3}
\end{array}\right)\left(\begin{array}{l}
v_{1} \\
v_{2} \\
v_{3}
\end{array}\right) .
\end{gathered}
$$

We then substitute these equations into the above defined system. For the function $w$, we use the three shape functions as the three test functions, which are necessary to solve for three unknowns. That is,

$$
w=\left(\begin{array}{l}
f_{1} \\
f_{2} \\
f_{3}
\end{array}\right) .
$$

Substituting these equations into our original system, we get the following system of integrals:

$$
\begin{aligned}
& \int_{\Omega_{e}}\left(\begin{array}{c}
f_{1} \\
f_{2} \\
f_{3}
\end{array}\right)\left(\begin{array}{c}
\dot{v}_{1} \\
\dot{v}_{2} \\
\dot{v}_{3}
\end{array}\right) d \Omega_{e}=D_{v} \int_{\Omega_{e}}\left(\left(\begin{array}{l}
\left(\frac{\partial f_{1}}{\partial x^{x}}\right. \\
\frac{\partial f_{2}}{\partial f_{3}^{2}} \\
\frac{\partial f_{3}}{\partial x}
\end{array}\right)\left(\begin{array}{lll}
\frac{\partial f_{1}}{\partial x} & \frac{\partial f_{2}}{\partial x} & \frac{\partial f_{3}}{\partial x}
\end{array}\right)\left(\begin{array}{c}
v_{1} \\
v_{2} \\
v_{3}
\end{array}\right)+\left(\begin{array}{c}
\frac{\partial f_{1}}{\partial y} \\
\frac{\partial f_{2}}{\partial y} \\
\frac{\partial f_{3}}{\partial y}
\end{array}\right)\left(\begin{array}{lll}
\frac{\partial f_{1}}{\partial y} & \frac{\partial f_{2}}{\partial y} & \frac{\partial f_{3}}{\partial y}
\end{array}\right)\left(\begin{array}{c}
v_{1} \\
v_{2} \\
v_{3}
\end{array}\right)\right) d \Omega_{e}
\end{aligned}
$$

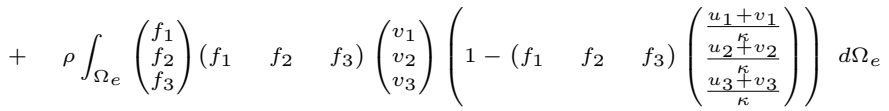

$$
\begin{aligned}
& \text { - } \beta \int_{\Omega_{e}}\left(\begin{array}{l}
f_{1} \\
f_{2} \\
f_{3}
\end{array}\right)\left(\begin{array}{lll}
f_{1} & f_{2} & f_{3}
\end{array}\right)\left(\begin{array}{l}
u_{1} \\
u_{2} \\
u_{3}
\end{array}\right) d \Omega_{e} \\
& \int_{\Omega_{e}}\left(\begin{array}{l}
f_{1} \\
f_{2} \\
f_{3}
\end{array}\right)\left(\begin{array}{l}
\dot{u}_{1} \\
u_{2} \\
u_{3}
\end{array}\right) d \Omega_{e}=D_{u} \int_{\Omega_{e}}\left(\left(\begin{array}{l}
\frac{\partial f_{1}}{\partial x} \\
\frac{\partial f_{2}}{\partial x} \\
\frac{\partial f_{3}}{\partial x}
\end{array}\right)\left(\begin{array}{lll}
\frac{\partial f_{1}}{\partial x} & \frac{\partial f_{2}}{\partial x} & \frac{\partial f_{3}}{\partial x}
\end{array}\right)\left(\begin{array}{l}
u_{1} \\
u_{2} \\
u_{3}
\end{array}\right)+\left(\begin{array}{c}
\frac{\partial f_{1}}{\partial y} \\
\frac{\partial f_{2}}{\partial y} \\
\frac{\partial f_{3}}{\partial y}
\end{array}\right)\left(\begin{array}{lll}
\frac{\partial f_{1}}{\partial y} & \frac{\partial f_{2}}{\partial y} & \frac{\partial f_{3}}{\partial y}
\end{array}\right)\left(\begin{array}{l}
u_{1} \\
u_{2} \\
u_{3}
\end{array}\right)\right) d \Omega_{e} \\
& +\beta \int_{\Omega_{e}}\left(\begin{array}{l}
f_{1} \\
f_{2} \\
f_{3}
\end{array}\right)\left(\begin{array}{lll}
f_{1} & f_{2} & f_{3}
\end{array}\right)\left(\begin{array}{l}
u_{1} \\
u_{2} \\
u_{3}
\end{array}\right) d \Omega_{e} .
\end{aligned}
$$

Simplifying somewhat, we get the linear system

$$
\begin{aligned}
{\left[M_{e}\right][\dot{v}]^{t} } & =D_{v}\left[K_{e}\right][v]^{t}-\rho\left[M_{e}\right]\left[v\left(1-\frac{v+u}{\kappa}\right)\right]^{t}-\beta\left[M_{e}\right][u]^{t} \\
{\left[M_{e}\right][\dot{u}]^{t} } & =D_{u}\left[K_{e}\right][u]^{t}+\beta\left[M_{e}\right][u]^{t}
\end{aligned}
$$


where the matrices are defined as

$$
\begin{aligned}
& {\left[M_{e}\right]=} \int\left(\begin{array}{lll}
f_{1} f_{1} & f_{1} f_{2} & f_{1} f_{3} \\
f_{2} f_{1} & f_{2} f_{2} & f_{2} f_{3} \\
f_{3} f_{1} & f_{3} f_{2} & f_{3} f_{3}
\end{array}\right) \\
& {\left[K_{e}\right]=\int\left(\begin{array}{lll}
\left(\frac{\partial f_{1}}{\partial x}\right)^{2}+\left(\frac{\partial f_{1}}{\partial y}\right)^{2} & \frac{\partial f_{1}}{\partial x} \frac{\partial f_{2}}{\partial x}+\frac{\partial f_{1}}{\partial y} \frac{\partial f_{2}}{\partial y} & \frac{\partial f_{1}}{\partial x} \frac{\partial f_{3}}{\partial x}+\frac{\partial f_{1}}{\partial y} \frac{\partial f_{3}}{\partial y} \\
\frac{\partial f_{2}}{\partial x} \frac{\partial f_{1}}{\partial x}+\frac{\partial f_{2}}{\partial y} \frac{\partial f_{1}}{\partial y} & \left(\frac{\partial f_{2}}{\partial x}\right)^{2}+\left(\frac{\partial f_{2}}{\partial y}\right)^{2} & \frac{\partial f_{2}}{\partial x} \frac{\partial f_{3}}{\partial x}+\frac{\partial f_{2}}{\partial y} \frac{\partial f_{3}}{\partial y} \\
\frac{\partial f_{3}}{\partial x} \frac{\partial f_{1}}{\partial x}+\frac{\partial f_{3}}{\partial y} \frac{\partial f_{1}}{\partial y} & \frac{\partial f_{3}}{\partial x} \frac{\partial f_{2}}{\partial x}+\frac{\partial f_{3}}{\partial y} \frac{\partial f_{2}}{\partial y} & \left(\frac{\partial f_{3}}{\partial x}\right)^{2}+\left(\frac{\partial f_{3}}{\partial y}\right)^{2}
\end{array}\right) . }
\end{aligned}
$$

It is not hard to calculate, with a little simple calculus, the explicit forms of these matrices, in terms of $a, b$, and $c$. Generalizing to the entire domain, we construct Equations (16) and (17), where the entire shape and stiffness matrices $[M]$ and $[K]$ are given by

$$
\begin{aligned}
& {[M]=\sum_{e=1}^{n}\left[M_{e}\right]} \\
& {[K]=\sum_{e=1}^{n}\left[K_{e}\right] .}
\end{aligned}
$$

Note that addition is performed by adding the entries of each matrix corresponding to the same node. For a more thorough explanation and excellent examples, see $[9,11]$. 